\title{
Life in a UCC hub
}

By Tara Barry, a GDP who usually works in Benfleet, Essex and Sudbury, Suffolk

$\mathrm{F}$ or someone who has spent years sitting side by side to patients, whether filling their teeth or yanking them out, I am finding the term 'social distancing' particularly difficult in my current situation working at an Urgent Dental Care Centre (UCC) hub.

Previously my working week involved working at a busy NHS/mixed practice alongside a private practice. My NHS practice is now a designated UCC hub and the private practice has closed its doors completely and is only offering telephone triage: a stark contrast to the dentistry I am used to. However, I feel privileged to see two sides of the same coin.

Essex was one of the first regions to have a triaged service in operation and our UCC hub went live on 2 April. A typical day involves seeing and treating patients who have been triaged for urgent care, and providing advice over the phone. Urgent dental treatment can mean different things criteria they are advised on the telephone before they visit, what to do when they arrive at the UCC. Once the patients arrive at the centre they are told to wait in their car until the clinicians and nurses are prepped and ready. There is no written medical history nor written treatment plan. This is all discussed on the phone prior to the patient arriving at the practice and documented. The practice layout has been altered to ensure social distancing measures are adhered to. The previously bustling waiting room is long gone.

Our UCC is a 'cold' hub whereby we see non-COVID vulnerable or self-isolating patients. Each patient is given a one-hour slot, to allow treatment and appropriate disinfection between patients. Like all new procedures in these unprecedented times there were teething problems (excuse the pun) initially; of course it was no-one's fault, just new and difficult circumstances that everyone was trying

\section{'Like all new procedures in these unprecedented times there were teething problems initially...'}

to different individuals so it has been difficult to ensure we are seeing those patients who have severe problems that their own GDP can't treat. This mainly consists of patients with severe toothache, broken teeth which prevent patients being able to function and eat, and a few oral medicine/dental trauma queries thrown in for good measure. We soon realised this can be particularly hard when we are relying on the patient to try and work out which tooth/area the pain is coming from (over the phone) with potentially no radiographs and occasionally 'selfies' to help diagnose. If patients fulfil our urgent to adjust to. Word had got around that we were a hub and dentists were desperate to provide care for their patients and hoping we could help them directly, but everyone needed to adhere to the Standard Operating Procedures (SOP) document outlined by NHS England and sent to all practices on 31 March 2020. Each day is a learning curve but there have been massive improvements from regular feedback from everyone.

With limited PPE, we are unable to offer aerosol generating procedures (AGPs) and are therefore severely limited as to what we can offer. Forceps are our new

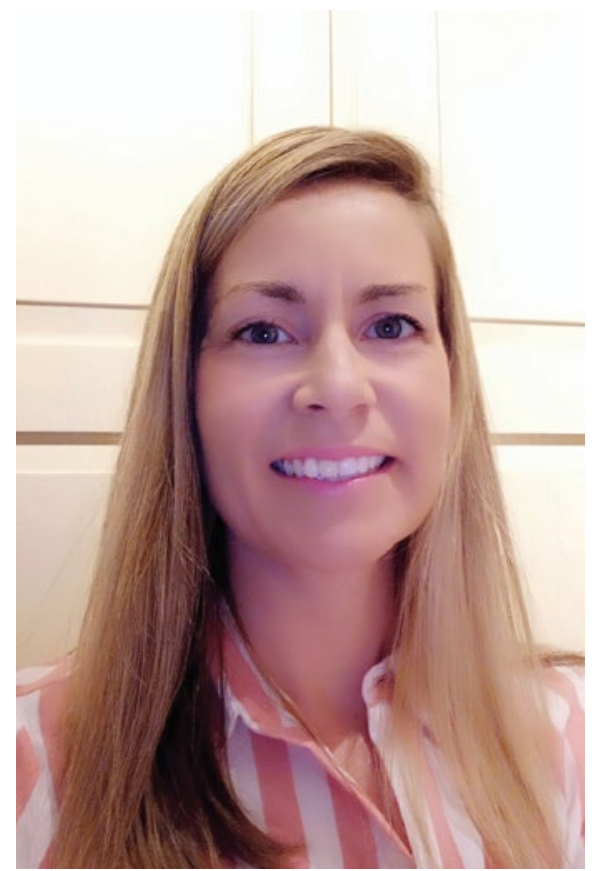

best friends. Dentists were referring for possible extirpations and we have no option currently but to offer extractions. This will hopefully be changing soon. Thus, treatment options for patients have been severely reduced. We currently have no means of removing impacted teeth or broken roots which would ordinarily have been removed by surgical means. I guess at this point we all realised our communication skills (and extraction skills) needed to be succinct and concise. Written patient consent seems a distant memory.

Meanwhile on the other side of the pond the private practice was receiving calls from our private patients since it closed its doors. Most of the calls are easy to manage following the three AAA (advice, analgesia and antimicrobials where appropriate), however I soon realised that DIY dentistry is going to be inevitable. An elderly gentleman who does not wish to be referred to a UCC as he is social distancing with his wife telephoned for advice to help remove his broken tooth. I've realised that telephone triaging can mean that diagnosing is difficult (it can be difficult at the best of times with the patient in front of us!) but I guess we use our 
4 experience and clinical judgement to do what is best for the patient given the current circumstances. Thankfully the patient didn't undertake DIY extraction (phew) and is extremely grateful for our advice and that 'we are only a phone call away'. Other calls involve our practice plan patients who are enquiring about their monthly practice payments. We try and reassure them that we are available 24/7 for advice but it's difficult for everyone. We are doing our best and trying to deliver our private prescriptions (appropriately) directly to patients' doors for the most vulnerable.

Patients always say to me they don't have time to floss but guess what, now IS the time to floss! There can be no better time to practise preventative dentistry when we are now limited to how we can treat patients.

Our NHS practice has also had to adapt to teaching our two foundation dentists (FDs) remotely. Both FDs were coming to the final months of their FD year. Ordinarily this is the time they would be starting to pick up speed and confidence; instead, their practical skills have come to a standstill. It can be a daunting time for them as their precious FD year has been robbed from them too. Instead though they have been shadowing/assisting us and we have been organising both theoretical and practical tutorials via Zoom. These carry with them to future employment and also life experiences. They have seen us utilise many emotional and practical skills from organisation and analytical skills to teamwork and lateral thinking.

I am immensely proud to be one of the team working at the UCC hub. Each and every person involved has worked tirelessly

\section{'Patients always say to me they don't have time to floss but guess what, now IS the time to floss!'}

are unprecedented times; usually we teach our FDs the importance of written consent and the need to discuss all the treatment options with the patients. Currently this has gone. I have been telling the FDs that witnessing this experience is something that cannot be taught. Being part of the UCC hub is invaluable experience they can to help develop and improve the system. Everyone has stepped up to the challenge and colleagues have been exceedingly flexible with working hours when childcare and family needs must be met. In these unprecedented times it makes me very proud to be a member of the dental profession. A big thank you to everyone. 